\title{
Maternal Exposure to Low Levels of Corticosterone during Lactation Protects the Adult Offspring against Ischemic Brain Damage
}

\author{
Paola Casolini, ${ }^{1}$ Maria Rosaria Domenici, ${ }^{2}$ Carlo Cinque, ${ }^{1}$ Giovanni Sebastiano Alemà, ${ }^{1}$ Valentina Chiodi, ${ }^{1}$ \\ Mariangela Galluzzo, ${ }^{2}$ Marco Musumeci, ${ }^{2}$ Jerome Mairesse, ${ }^{3}$ Anna Rita Zuena, ${ }^{1}$ Patrizia Matteucci, ${ }^{1}$ Giuseppe Marano, ${ }^{2}$ \\ Stefania Maccari, ${ }^{3,5}$ Ferdinando Nicoletti, ${ }^{1,4}$ and Assia Catalani ${ }^{1}$ \\ ${ }^{1}$ Department of Human Physiology and Pharmacology, University of Rome "La Sapienza," Faculty of Medicine, 00185 Rome, Italy, ${ }^{2}$ Department of \\ Pharmacology, Istituto Superiore di Sanità, 00161 Rome, Italy, ${ }^{3}$ Laboratory of Perinatal Stress, University of Lille 1, 59655 Villeneuve d’Ascq, France, \\ ${ }^{4}$ Istituto Neurologico Mediterraneo, Neuromed Pozzilli, 86077 Pozzilli, Italy, and ${ }^{5}$ University of Rome "La Sapienza," Faculty of Medicine, 00185 Rome, \\ Italy
}

A growing body of evidence underscores the importance of early life events as predictors of health in adulthood. Abnormalities in maternal care or other forms of early postnatal stress induce long-term changes in behavior and influence the vulnerability to illnesses throughout life. Some of these changes may be produced by the activation of the hypothalamic-pituitary-adrenal (HPA) axis, which is invariably associated with stress. We used a model in which neonate rats are fed by mothers drinking water supplemented with $0.2 \mathrm{mg} / \mathrm{ml}$ corticosterone, the main glucocorticoid hormone in rodents. Plasma corticosterone levels increased in the dams to an extent similar to that induced by a mild stress. Corticosterone-treated dams also showed an increase in maternal care. Remarkably, adult rats that had been nursed by corticosterone-treated mothers were protected against neuronal damage and cognitive impairment produced by transient global brain ischemia. Neuroprotection was associated with a reduced HPA response to ischemia and was primarily decreased when corticosterone was injected at a dose that eliminated any difference in endogenous corticosterone levels between rats raised by mothers supplemented with corticosterone and their matched controls. These data suggest that an increased maternal care protects the offspring against ischemic neuronal damage and that at least a component of neuroprotection is mediated by a reduced response of the HPA axis to ischemia.

Key words: corticosteroids; early events in life; brain damage; neuronal vulnerability; ischemia; stress

\section{Introduction}

Early-life environmental factors may cause structural and functional changes in the CNS, which persist for the entire lifespan and affect synaptic plasticity, behavior, and responses to stress. For example, changes in maternal care produce permanent alterations in the hypothalamic-pituitary-adrenal (HPA) response to stress, which reflect a long-lasting experience-dependent form of chromatin plasticity in the hippocampus (Weaver et al., 2004; Meaney and Szyf, 2005; Kaffman and Meaney, 2007). Similar changes in the HPA axis are found in adult rats that had been exposed to mild levels of corticosterone (the main glucocorticoid hormone in rodents) during lactation. These rats show a blunted HPA response to restraint stress because of a more efficient negative feedback mediated by glucocorticoid receptors in the hippocampus (Casolini et al., 1997; Catalani et al., 2000). Glucocor-

Received Aug. 9, 2006; revised May 17, 2007; accepted May 22, 2007

This study was supported by grants from the University of Rome "La Sapienza."

Correspondence should be addressed to Assia Catalani, Department of Human Physiology and Pharmacology, University of Rome "La Sapienza," Piazzale Aldo Moro 5, 00185 Rome, Italy. E-mail: assia.catalani@uniroma1.it. DOI:10.1523/JNEUROSCI.1074-07.2007

Copyright $\odot 2007$ Society for Neuroscience $\quad$ 0270-6474/07/277041-06\$15.00/0 ticoids bind to two cytoplasmic receptor subtypes: (1) mineralcorticoid receptors (MRs), which are mainly expressed in limbic regions and bind corticosterone with high affinity $\left(K_{\mathrm{D}}\right.$ of $0.5 \mathrm{nM}$ ) and (2) glucocorticoid receptors (GRs), which are ubiquitous and bind corticosterone with a 10 -fold lower affinity $\left(K_{\mathrm{D}}\right.$ of $5 \mathrm{nM}$ ). The different affinities of MRs and GRs account for the typical inverse- $U$ profile of responses to glucocorticoids in a wide range of domains (for review, see De Kloet et al., 1998, 2007). Interestingly, activation of MRs and GRs differentially affects neuronal viability (see Discussion and references therein). Because a sustained activation of the HPA axis may be harmful to CNS neurons (Sapolsky, 1986; McEwen, 1999; Abraham et al., 2001), we predicted that an early-life exposure to glucocorticoids could result into permanent changes in neuronal vulnerability. Transient global ischemia in rats is one of the situations in which damage of vulnerable neurons depends on the extent of activation of the HPA axis. Ischemic neuronal death is potentiated by exposure to high physiological titers of glucocorticoids and is attenuated by adrenalectomy (Sapolsky and Pulsinelli, 1985). We now report that the adult offspring of mothers that received corticosterone during lactation ("CORT-nursed" rats) were pro- 
tected against neuronal damage produced by transient global brain ischemia.

\section{Materials and Methods}

The CORT-nursed model. Female Wistar rats (Charles River, Calco, Italy) weighing $280-320 \mathrm{~g}$ were mated and then housed individually. The day of birth was counted as day 0 , and the next day litters were culled to eight pups (four males and four females). Mothers of control rats were maintained on tap water, whereas mothers of CORT-nursed rats had ad libitum access to a solution of $0.2 \mathrm{mg} / \mathrm{ml}$ corticosterone hemisuccinate. The average daily intake of corticosterone was $\sim 13-15 \mathrm{mg} / \mathrm{rat}$. Weaning was performed at $21 \mathrm{~d}$ of age, and animals were then housed three per cage. No difference in body and adrenal weights was found between adult CORT-nursed and control rats. Three-month-old male CORT-nursed rats and their controls were used in this study.

Assessment of maternal behavior. The behavior of each dam was observed five times per day ( $60 \mathrm{~min}$ each; three times during the light phase and two times during the dark phase of the day) from day 2 to 11 after delivery. We recorded the frequency of maternal licking and grooming (LG) of pups and arched-back nursing (ABN).

Corticosterone secretion during the light and the dark period of the diurnal cycle and after a restraint stress. Blood was drawn from the tip of the tail $(300 \mu \mathrm{l}$ of blood in $2 \mathrm{mg} / \mathrm{ml}$ EDTA, time taken to collect less then 2 $\mathrm{min}$ ) at 8:00 AM, 8:00 PM, and, in different groups of animals, at 0, 15, and $90 \mathrm{~min}$ from $2 \mathrm{~min}$ restraint stress. After centrifugation at $1900 \times \mathrm{g}$ at $4^{\circ} \mathrm{C}$ for $15 \mathrm{~min}$, plasma was removed and kept frozen at $-20^{\circ} \mathrm{C}$.

Corticosterone assay. Plasma corticosterone concentrations were determined by radioimmunoassay (MP Biomedicals, Strasbourg, France). The cross-reactivity of the polyclonal corticosterone antisera with respective related substances was negligible. The interassay and intraassay coefficients of variance were 7 and $4 \%$, respectively, with a detection limit of $0.01 \mu \mathrm{g} / 100 \mathrm{ml}$.

Induction of transient global ischemia. Transient global ischemia was induced by combining bilateral carotid artery occlusion with hypotension (Ferrand-Drake and Wieloch, 1999), as follows. In a first group of 16 adult CORT-nursed rats and their age-matched controls, animals were anesthetized with Equithesin $(3 \mathrm{ml} / \mathrm{kg}$, i.p. $)$, and the right femoral artery was cannulated to allow monitoring of arterial blood pressure. Body temperature was maintained at $37^{\circ} \mathrm{C}$ throughout the procedure with a feedback-regulated heat pad (Harvard homeothermic blanket control unit, 50-7061; Harvard Apparatus, Holliston, MA). The common carotid arteries were isolated, and blood pressure was lowered with a subcutaneous injection of sodium nitroprusside $(2.5 \mathrm{mg} / \mathrm{kg})$. When blood pressure was reduced to $50 \mathrm{mmHg}$, both carotid arteries were clamped with nontraumatic aneurysm clips for $10 \mathrm{~min}$. Eight ischemic CORT-nursed and controls rats were used for behavioral and histological analysis. The remaining rats either died or did not reach the threshold of $50 \mathrm{mmHg}$ in response to nitroprusside. Seven sham-operated CORT-nursed rats and eight controls underwent surgery without carotid occlusion.

Additional groups of sham-operated or ischemic CORT-nursed rats and their respective controls were either treated with saline or with corticosterone hemisuccinate $(0.25 \mathrm{mg} / \mathrm{kg}$, s.c. $)$ at the time of carotid artery occlusion in ischemic rats or the corresponding time in sham-operated rats. All of these animals were used for the assessment of plasma corticosterone levels and neuronal death. Corticosterone levels were measured $24 \mathrm{~h}$ before surgery, $25 \mathrm{~min}$ after surgery in sham-operated rats or $15 \mathrm{~min}$ after reperfusion in ischemic rats, and $24 \mathrm{~h}$ after surgery or ischemia. Neuronal death was assessed $7 \mathrm{~d}$ after ischemia (or surgery in shamoperated rats).

Assessment of spatial learning. Cognitive performance was assessed in the Morris water maze as described by Catalani et al. (1993). Acquisition consisted of five trials per day for 3 consecutive days. After the last trial of the last day of training, animals were submitted to a single $60 \mathrm{~s}$ "probe trial."

Histological processing. Anesthetized (pentobarbital $60 \mathrm{mg} / \mathrm{kg}$ ) animals were transcardially perfused with saline, followed by fixation with $4 \%$ paraformaldehyde dissolved in $0.1 \mathrm{M}$ phosphate buffer. Brains were removed from the skull, postfixed overnight at $4^{\circ} \mathrm{C}$ in buffered $4 \%$ paraformaldehyde, and stored at $4^{\circ} \mathrm{C}$ in $20 \%$ sucrose solution until they sank.
Brains were frozen, and coronal $10 \mu \mathrm{m}$ sections at the level of the hippocampal formation were cut on a microtome cryostat and mounted on Superfrost Plus microscope slide. Sections were Nissl stained with $0.2 \%$ thionin (Merck, Darmstadt, Germany) in acetate buffer for $8 \mathrm{~min}$ and dehydrated through graded alcohols (70, 95, and 100\%) and in xylene; finally, they are coverslipped using Entellan (Merck).

Assessment of neuronal damage. Cell counts of CA1 and CA3 subfields neurons were performed at three levels of the dorsal hippocampus. Specifically, sections were obtained at 3.2,3.7, and $4.2 \mathrm{~mm}$ posterior to the bregma; both left and right hemisphere from each level were used $(n=6$ regions in both CA1 an CA3 regions for each animal). The number of viable neurons (that did not show pyknosis or shrinkage) within the pyramidal layer were counted using a light microscope (model BH-2; Olympus Optical, Tokyo, Japan) at a magnification of $200 \times$. Counts are expressed as the number of CA1 and CA 3 neurons per millimeter of linear length as described previously (Choi et al., 2005) with some modifications. To maintain consistency across animals, a rectangular box $(0.5 \times 0.3 \mathrm{~mm})$ was centered over the CA1 pyramidal layer beginning 2.0 $\mathrm{mm}$ lateral to the midline, and another similar box was placed in the corner of CA3 subfield pyramidal layer. Analyses were performed with the aid of NIH Image software. Neurons were counted by two observers who were unaware of the experimental protocol.

Statistical analysis. Statistical analysis was performed by two-way or one-way ANOVA with post hoc Fisher's least significant difference (LSD), ANOVA for repeated measures, or by Student's $t$ test, as indicated in the figure legends.

\section{Results}

We used 3-month-old male rats that had been exposed to mild levels of corticosterone through maternal milk during the whole lactation period (first $21 \mathrm{~d}$ of postnatal life). Plasma corticosterone levels assessed in a parallel group of 10-d-old CORT-nursed pups were doubled with respect to control pups that did not receive corticosterone through the maternal milk ( $1.1 \pm 0.09$ and $0.58 \pm 0.06 \mu \mathrm{g} / 100 \mathrm{ml}$, respectively, means $\pm \mathrm{SEM} ; n=10 ; p<$ 0.05 , Student's $t$ test). The weight of adrenal glands was also reduced in these representative CORT-nursed pups with respect to their controls $(3.9 \pm 0.18$ and $6.1 \pm 1.2 \mathrm{mg}$, respectively). Body weight did not differ between control and CORT-nursed pups (Catalani et al., 1993). Plasma corticosterone levels in representative mothers of CORT-nursed rats increased from $5.7 \pm 0.4$ to $13 \pm 0.9 \mu \mathrm{g} / 100 \mathrm{ml}$ at day 10 of lactation (means $\pm \mathrm{SEM} ; n=5$; $p<0.05$, Student's $t$ test)

We also examined whether corticosterone supply to dams influenced maternal behavior for the following reasons: (1) corticosterone treatment in adrenalectomized primiparous rats increases maternal behavior in a dose-dependent manner (Rees et al., 2004), and (2) maternal care plays an important role in the CNS development of the offspring (Weaver et al., 2004; Meaney and Szyf, 2005; Kaffman and Meaney, 2007). Mothers of CORTnursed rats showed an increased care of their pups, as assessed by measurements of licking/grooming arched-back nursing. Mothers of CORT-nursed rats showed an increased frequency of licking/grooming of their pups during the whole period tested (from day 2 to 11 after delivering) (Fig. $1 A$ ). The increased frequency of arched-back nursing was significant when examined from day 5 to 11 after delivery, i.e., after the initial period of intense archedback nursing (Fig. $1 B$ ).

We used exclusively male CORT-nursed rats because there is a gender effect in the neuroendocrine profile of adult CORTnursed rats (Catalani et al., 2000, 2002) and because ovarian hormones influence neuronal vulnerability to ischemic damage (Hoffman et al., 2006). Adult CORT-nursed rats show changes in the activity of the HPA axis, as reflected by a reduced response of corticosterone to stress (Catalani et al., 2000). This likely results 


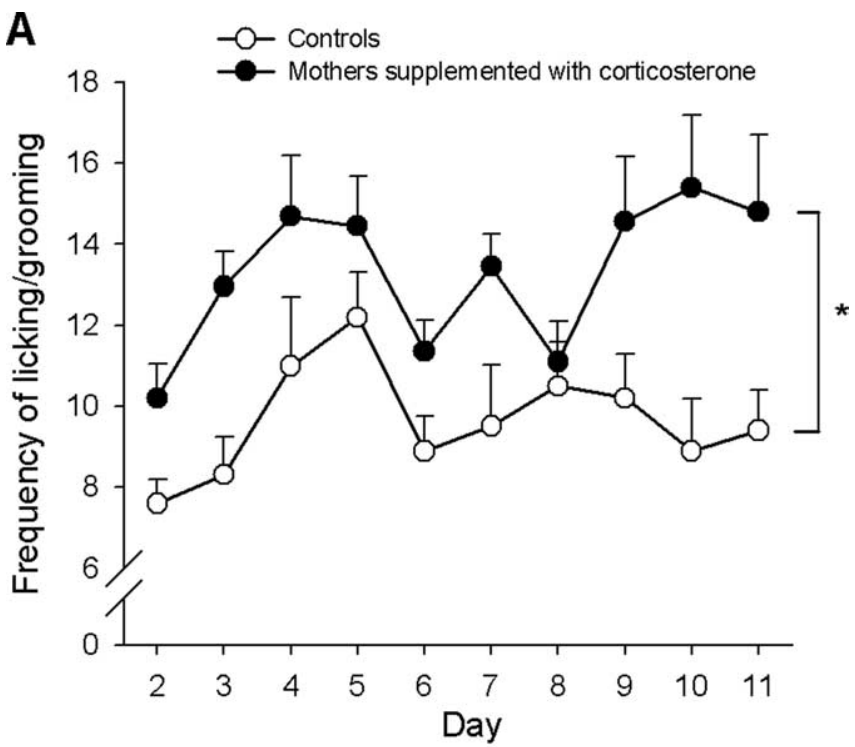

B

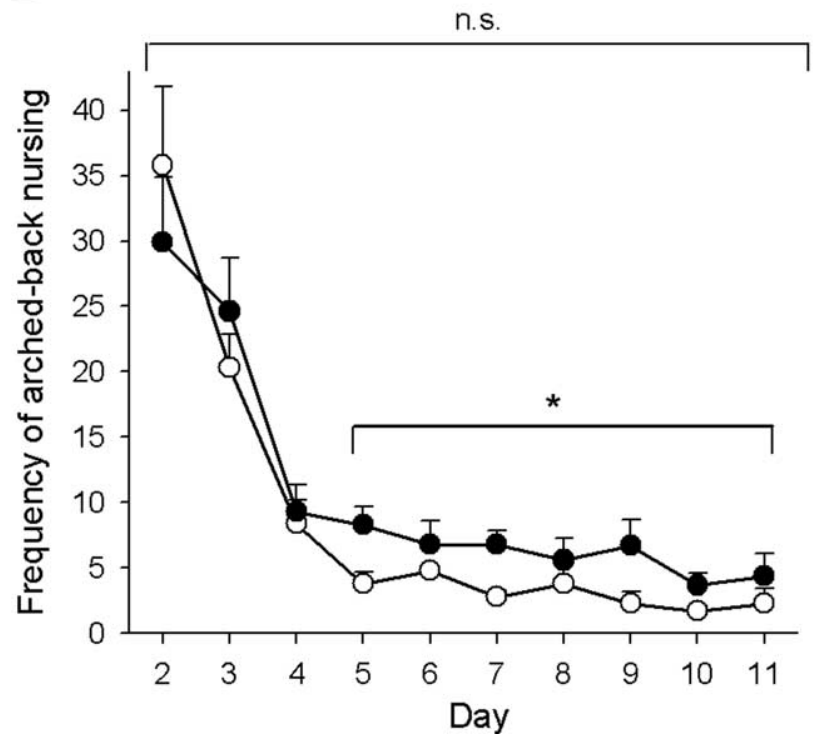

Figure 1. Increased maternal behaviors in dams drinking water supplemented with corticosterone (mothers of CORT-nursed rats). The frequency of licking/grooming of control mothers and mothers of CORT-nursed rats from day 2 to 11 after delivery (observed during 5 60-min periods per day) is shown in $\boldsymbol{A}$. Values are means \pm SEM of 10 mothers per group. ${ }^{*} p<0.05$ (ANOVA for repeated measures) versus control mothers. The frequency of arched-back nursing in the same periods is shown in $\boldsymbol{B}$. The difference between mothers of CORT-nursed rats and control mothers was significant when evaluated from day 5 to 11 after delivery ${ }^{*} p<0.05$ ANOVA for repeated measures) but was nonsignificant (n.s.) when evaluated during the whole observation period (from day 2 to 11 after delivery).

from an increased expression of both MRs and GRs in the hippocampus, as shown by radioligand binding analysis in CORTnursed rats of 1 and 15 months of age (Casolini et al., 1997; Catalani et al., 2000). An increase in MR and GR density ( $24 \pm 3.7$ and $14 \pm 4.5 \%$ vs controls, respectively; $n=5)$ is also observed in the hippocampus of 2-month-old CORT-nursed rats (our unpublished observation). Here, we could not examine the density of MRs and GRs in the hippocampus of CORT-nursed rats because selective radioactive receptor ligands are no longer available. However, we examined the activity of the HPA axis in our 3-month-old CORT-nursed rats by assessing plasma corticoste-

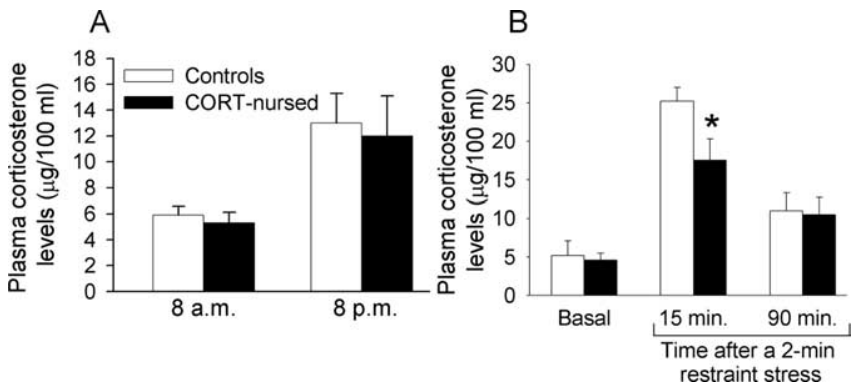

Figure 2. Reduced HPA response to restraint stress in adult rats that had been exposed to mild levels of corticosterone during lactation (CORT-nursed rats). Basal plasma corticosterone levels in the light (8:00 AM) and dark (8:00 PM) period of the diurnal cycle of control and CORT-nursed rats are shown in $\boldsymbol{A}$. Light was turned on at 7:00 AM and turned off at 7:00 PM. Values are means \pm SEM of determinations of seven to eight animals. Corticosterone levels in CORT-nursed and control rats subjected to a 2 min restraint stress are shown in $\boldsymbol{B}$. Values are means \pm SEM of determinations of six animals. ${ }^{*} p<0.05$ versus the respective controls (Student's $t$ test).

rone levels under basal conditions at 8:00 A.M. and 8:00 P.M., as well as in response to a $2 \mathrm{~min}$ restraint stress. Although we found no difference in basal corticosterone levels (Fig. $2 \mathrm{~A}$ ), the corticosterone response to stress was blunted in CORT-nursed rats (Fig. $2 B$ ), as expected (Catalani et al., 2000). Thus, we confirmed the lower reactivity of the HPA axis to stress in our CORT-nursed rats.

CORT-nursed rats and their age-matched controls underwent transient global ischemia by bilateral carotid artery occlusion combined with a severe fall in blood pressure $(<50 \mathrm{mmHg})$ (Ferrand-Drake and Wieloch, 1999). Four days after surgery, ischemic and sham-operated rats were tested for 3 consecutive days in the Morris water maze for the assessment of spatial learning, a task that is highly sensitive to hippocampal damage. Shamoperated CORT-nursed rats performed better than shamoperated control rats on days 2 and 3 of training, as expected (Catalani et al., 1993) (Fig. 3A,B). A remarkable difference in spatial learning was found between ischemic CORT-nursed and control rats. Whereas ischemic controls showed a severe learning deficit, ischemic CORT-nursed rats learned similarly to shamoperated rats (Fig. $3 A, B$ ). When the platform was made visible, there were no group differences (all latencies $<10$ s), indicating the absence of sensory motor deficits.

Soon after the end of the last training session, animals were killed, and brain sections were Nissl stained and assessed for neuronal death using a nonstereological but reliable method of cell counting (Choi et al., 2005). In control rats, global ischemia induced a substantial neuronal loss (95\%) in the CA1 region without affecting neuronal viability in the CA3 region (Fig. $3 C-E$ ). Early-life exposure to corticosterone was protective against ischemic neuronal damage, although a significant damage was still observed with respect to sham-operated rats $(\sim 40 \%$ of neuronal loss in the CA1 neurons $7 \mathrm{~d}$ after ischemia) (Fig. $3 C-E$ ).

To unravel the mechanism that could protect CORT-nursed rats against ischemic damage, we assessed corticosterone levels $1 \mathrm{~d}$ before ischemia (indicated as "basal") and $15 \mathrm{~min}$ and $24 \mathrm{~h}$ after reperfusion. Corticosterone levels were also assessed in parallel groups of sham-operated rats that underwent surgery without the induction of ischemia. In both ischemic and shamoperated control rats (i.e., rats that were not CORT-nursed), surgery induced a substantial rise in corticosterone levels after 25 min (i.e., 25 min after surgery in sham-operated rats and $15 \mathrm{~min}$ after reperfusion in ischemic rats). In both groups of rats, corti- 
costerone levels slightly declined but remained high at $24 \mathrm{~h}$. The increase in corticosterone levels was substantially attenuated in both ischemic and shamoperated CORT-nursed rats at $25 \mathrm{~min}$. In contrast, no difference was found between CORT-nursed and control rats at $24 \mathrm{~h}$ (Fig. $4 A$ ). In an attempt to eliminate any difference between corticosterone levels between CORT-nursed and control rats, two groups of animals were treated subcutaneously with $0.25 \mathrm{mg} / \mathrm{kg}$ corticosterone hemisuccinate at the time of carotid artery occlusion in ischemic rats or at the time of surgery in sham-operated rats. This treatment produced only small increases in corticosterone levels in ischemic and sham-operated control rats (Fig. 4A) but substantially enhanced corticosterone levels of ischemic or sham-operated CORTnursed rats at $25 \mathrm{~min}$. After treatment with corticosterone hemisuccinate, CORTnursed and control rats showed similar corticosterone levels at $25 \mathrm{~min}$ (Fig. 4A).

None of the control or CORT-nursed sham-operated rats treated with corticosterone hemisuccinate died. In addition, corticosterone treatment in these animals did not produce any cell loss in the hippocampus at $7 \mathrm{~d}$ after surgery (Fig. $4 B$ ). Control ischemic rats treated with corticosterone hemisuccinate showed the same rate of mortality as control ischemic rats treated with saline (5 of 10 and 4 of 8 animals, respectively). The extent of cell death in the hippocampal CA1 regions was nearto-maximal in both groups of animals. CORT-nursed ischemic rats treated with corticosterone showed a high mortality rate with respect to CORT-nursed ischemic rats treated with saline (6 of 11 and 1 of 6 animals, respectively). The extent of CA1 neuronal death in surviving CORTnursed ischemic rats treated with corticosterone hemisuccinate was significantly higher than in CORT-nursed ischemic rats treated with saline, although it was still lower than that observed in control ischemic rats (i.e., in rats that had not been exposed to corticosterone during lactation) (Fig. $4 B$ ). No neuronal damage was found in the CA3 region in any of the experimental groups (data not shown).

\section{Discussion}

We found that administration of mild levels of corticosterone to lactating mothers results into a decreased vulnerability of hippocampal neurons to ischemic lesion in the adult progeny and that much of this effect can be attributed to a lower reactivity of the HPA axis. This is consistent with the evidence that neonatal handling, which also leads to a long-lasting hyporeactivity of the HPA axis (Bhatnagar and Meaney, 1995; Vallèe et al., 1997), attenuates the spontaneous loss of hippocam-

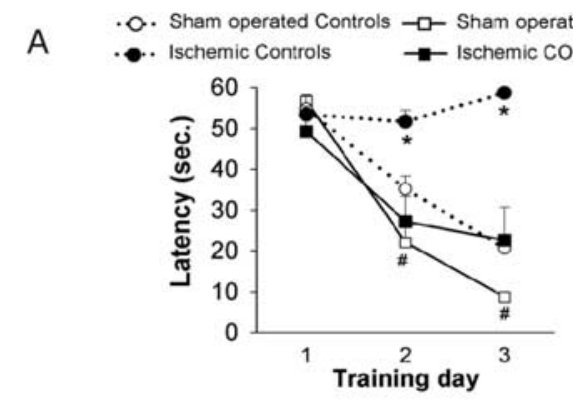

Sham operated rats
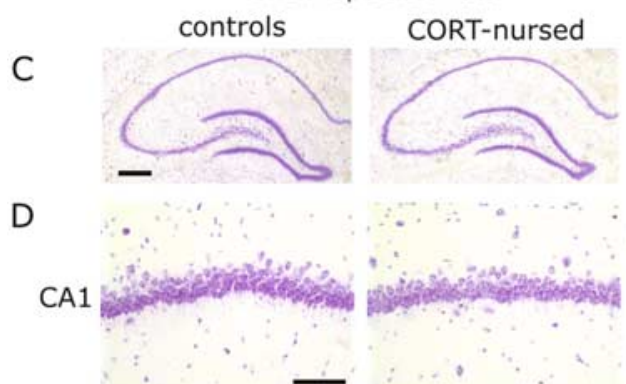

CA3

E

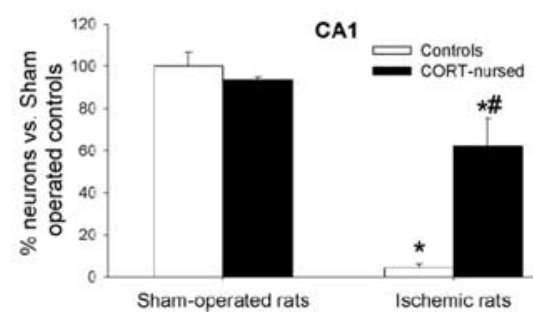

B

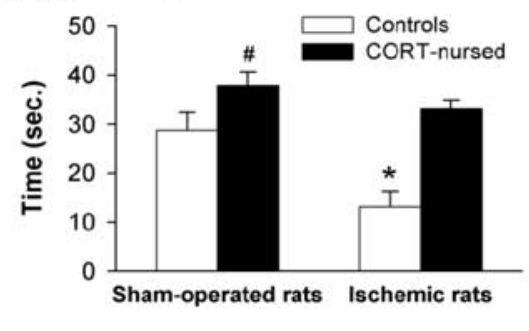

Ischemic rats

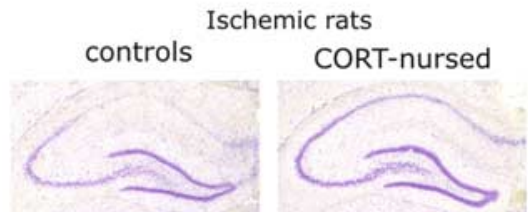

Figure 3. Adult CORT-nursed rats are protected against hippocampal neuronal damage induced by transient global cerebral ischemia. Data obtained with the Morris water maze in sham-operated and ischemic control or CORT-nursed rats are shown in $\boldsymbol{A}$ and $\boldsymbol{B}$. Spatial learning was assessed $4 \mathrm{~d}$ after ischemia (or the corresponding days after surgery in sham-operated rats). $\ln \boldsymbol{A}$, values are means \pm SEM of five trials per day for 3 training days from seven to eight animals. There was a significant group effect and a significant group $\times$ day interaction effect. One-way ANOVA performed separately on each day showed a significant group difference on days 2 and 3 . In ischemic animals, the control group was unable to learn the task ( ${ }^{*} p<0.05$ vs all other groups), whereas the performance of ischemic CORT-nursed was similar to that of sham-operated animals. Sham-operated CORT-nursed rats performed better than sham-operated controls, as expected ( ${ }^{\#} p<0.05$, post hoc Fisher's LSD). Data in $\boldsymbol{B}$ show that both sham-operated and ischemic CORT-nursed rats spent more time in the quadrant in which the hidden platform was present before being removed from the maze ("probe trial"). Values are means \pm SEM of seven to eight determinations. ${ }^{*} p<0.05$ versus all other groups; $" p<0.05$ versus matched controls (one-way ANOVA with Fisher's LSD). Niss staining of representative hippocampi from sham-operated and ischemic CORT-nursed rats and their controls is shown in C. Scale bar, $500 \mu \mathrm{m}$. A higher magnification of a selected area in the CA1 and CA3 regions is shown in $\boldsymbol{D}$. Scale bar, $100 \mu \mathrm{m}$. In sham-operated animals, the average counts of pyramidal neurons were $201 \pm 7.8$ and $150 \pm 2.5 / \mathrm{mm}$ in the CA1 and CA3 subfields, respectively. In $\boldsymbol{E}$, neuronal loss in the CA1 region was much lower in ischemic CORT-nursed rats than in ischemic controls. No changes in neuronal counts were found in the CA3 regions. Values are expressed as percentage of sham-operated animals and represent the means \pm SEM of seven to eight determinations. ${ }^{*} p<0.05$ versus the respective groups of sham-operated rats; ${ }^{*} p<0.05$ versus the respective control rats (one-way ANOVA with Fisher's LSD).

pal neurons in senescent rats (Meaney et al., 1988). Thus, early environmental manipulations might contribute to set the threshold for neuronal death under physiological and pathological conditions. An important issue to be discussed is why CORT-nursed rats develop a hyporeactivity of the HPA axis. A series of elegant studies show that maternal care critically affects the development of the HPA axis in the progeny. For example, neonatal handling 


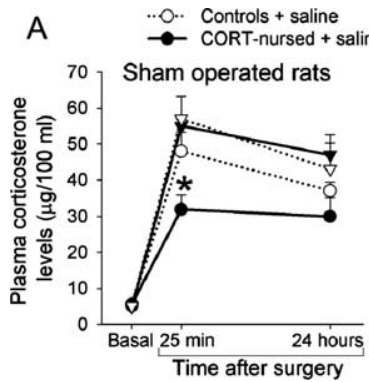

$\cdots \nabla \cdot$ Controls + Cort hem
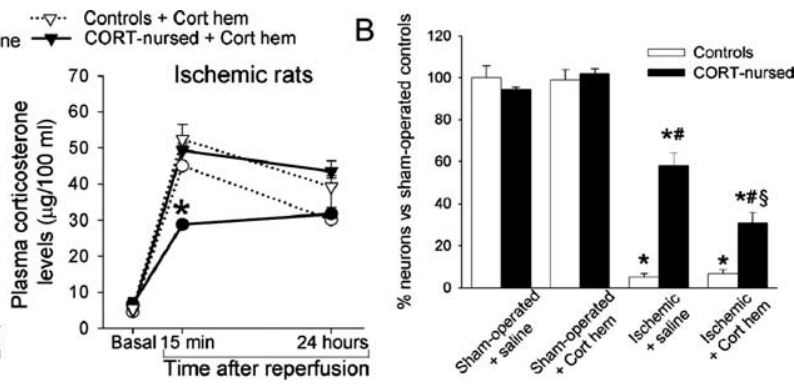

Figure 4. Plasma corticosterone levels and hippocampal neuronal damage in ischemic control and CORT-nursed rats treated with saline or with corticosterone hemisuccinate. In $A$, plasma corticosterone levels are shown in sham-operated and ischemic control or CORT-nursed rats treated with saline or corticosterone hemisuccinate (Cort hem) under basal conditions ( $24 \mathrm{~h}$ before surgery/ischemia) or at $25 / 15 \mathrm{~min}$ and $24 \mathrm{~h}$ after surgery/reperfusion. Neuronal counts in the CA1 region of all animals are shown in $\boldsymbol{B}$. Animals were treated with saline or corticosterone hemisuccinate at the time of carotid artery occlusion or at the corresponding time in sham-operated rats. Values are means \pm SEM of four to five determinations. In $\boldsymbol{A}^{*}{ }^{*} p<0.05$ (one-way ANOVA with Fisher's LSD) versus all other corresponding groups. $\ln \boldsymbol{B},{ }^{*} p<0.05$ (one-way ANOVA with Fisher's LSD) versus the corresponding sham-operated groups; ${ }^{\#} p<0.05$ versus the corresponding group of control rats; ${ }^{\circledR} p<0.05$ versus ischemic CORT-nursed rats treated with saline.

itself enhances maternal behavior, and the adult offspring of mothers that naturally display an increased licking and grooming of pups and arched-back nursing ("high LG/ABN mothers") shows a blunted HPA response to stress (Liu et al., 1997; Weaver et al., 2004; Meaney and Szyf, 2005; Kaffman and Meaney, 2007). The latter effect derives from specific epigenomic mechanisms that involve changes in the regulation of the GR gene in the hippocampus (Weaver et al., 2004; Meaney and Szyf, 2005). Mothers receiving corticosterone with the drinking water showed an increased frequency of LG of pups and $A B N$, similar to what found in adrenalectomized primiparous rats treated with corticosterone (Rees et al., 2004). Thus, it is reasonable to hypothesize that the increased maternal care is a major determinant of the lower reactivity of the HPA axis observed in adult CORT-nursed rats. However, we cannot exclude that the early exposure of pups to maternal corticosterone through the milk in a period of relative adrenocortical quiescence (i.e., in the first 2 weeks of postnatal life) (Sapolsky and Meaney, 1986) might have contributed to the induction of long-lasting changes in the HPA axis. Interestingly, both handled pups and CORT-nursed pups show double levels of corticosterone during lactation (Moles et al., 2004) (also see present data). However, it is noteworthy that direct injections of corticosterone to pups do not result into long-lasting alterations of the HPA axis (Meaney et al., 1987). Handled rats and CORT-nursed rats share a permanent hyporeactivity of the HPA axis associated with an increased GR density in the hippocampus, although subtle differences in the temporal profile of GR expression are found between the two models (Casolini et al., 1997; Liu et al., 1997; Avishai-Eliner et al., 2001). However, a difference is found in the expression of MRs in the hippocampus, which is not altered in handled rats (Meaney et al., 1992; Weaver et al., 2001) but is increased in CORT-nursed rats (Casolini et al., 1997). It will be interesting to examine whether handled rats or rats fed by high LG/ABN mothers show a lower neuronal vulnerability to ischemic insult.

CORT-nursed rats were strongly resistant to transient global cerebral ischemia, as shown by a lower extent of neuronal loss in the hippocampus and by the lack of learning impairment in the water maze. Two sets of data suggest that this resistance was primarily dependent on a hyporeactivity of the HPA axis: (1) the apparent peak in plasma corticosterone levels measured 25 min after surgery (i.e., $15 \mathrm{~min}$ after reperfusion) was substantially lower in CORT-nursed rats, and (2) corticosterone supply at a dose that eliminates the difference in peak corticosterone levels between CORT-nursed and control rats enhanced ischemic neuronal death in CORT-nursed rats. Our findings support the view that ischemic hippocampal damage depends on the extent of activation of the HPA axis (Sapolsky and Pulsinelli, 1985). A likely scenario is that GRs, which display a low affinity to corticosterone and are potentially harmful to hippocampal neurons (Koide et al., 1986; Abraham et al., 2000; Liu et al., 2005), are less activated in CORT-nursed rats because of the lower peak of circulating corticosterone. In addition, our data suggest that the permissive action of corticosterone occurs during the early induction phase of ischemic neuronal damage (e.g., 15 min after reperfusion), when we found the difference in corticosterone levels between control and CORT-nursed rats. However, we cannot precisely define the time window of the permissive action of corticosterone because we measured plasma corticosterone levels only at two times (i.e., $25 \mathrm{~min}$ and $24 \mathrm{~h}$ ) after ischemia.

Because CORT-nursed rats were still partially protected after treatment with corticosterone, it is possible that factors other than the lower reactivity of the HPA axis contribute to the resistance of these animals to ischemic damage. MRs, which are found at higher density in the hippocampus of CORT-nursed rats (Casolini et al., 1997), may protect hippocampal neurons against ischemic damage independently of their ability to regulate the HPA axis (Macleod et al., 2003). In addition, CORT-nursed rats show a lower expression of mGlul metabotropic glutamate receptors (Cinque et al., 2003), the activation of which contributes to the pathophysiology of ischemic hippocampal damage (De Vry et al., 2001; Moroni et al., 2002; Pellegrini-Giampietro, 2003).

In conclusion, our data suggest that manipulations of postnatal development modify neuronal vulnerability to ischemic lesions in adulthood primarily as a result of long-term alterations in HPA reactivity. CORT-nursed rats provide an example of how a particular manipulation of postnatal development leads to a persistent hyporeactivity of the HPA axis with an ensuing resistance to ischemic neuronal damage. Other manipulations, such as the administration of high doses of corticosterone throughout the perinatal period, result into a long-lasting hyperreactivity of the HPA axis (Felszeghy et al., 1996; Bakker et al., 1997), which may produce detrimental effects that may persist across life and are potentially life threatening (Felszeghy et al., 1996; Shinwell et al., 2000; Kamphuis et al., 2002).

\section{References}

Abraham I, Harkany T, Horvath KM, Veenema AH, Penke B, Nyakas C, Luiten PG (2000) Chronic corticosterone administration dosedependently modulates Abeta(1-42)-and NMDA-induced neurodegeneration in rat magnocellular nucleus basalis. J Neuroendocrinol $12: 486-494$.

Abraham IM, Harkany T, Horvath KM, Luiten PG (2001) Action of glucocorticoids on survival of nerve cells: promoting neurodegeneration or neuroprotection? J Neuroendocrinol 13:749-760.

Avishai-Eliner S, Eghbal-Ahmadi M, Tabachnik E, Brunson KL, Baram TZ (2001) Down-regulation of hypothalamic corticotropin-releasing hor- 
mone messenger ribonucleic acid (mRNA) precedes early-life experience-induced changes in hippocampal glucocorticoid receptor mRNA. Endocrinology 142:89-97.

Bakker JM, Schmidt ED, Kroes H, Kavelaars A, Heijnen CJ, Tilders FJ, van Rees EP (1997) Effects of neonatal dexamethasone treatment on hypothalamo-pituitary adrenal axis and immune system of the rat. J Neuroimmunol 74:69-76.

Bhatnagar S, Meaney MJ (1995) Hypothalamic-pituitary-adrenal function in chronic intermittently cold-stressed neonatally handled and non handled rats. J Neuroendocrinol 7:97-108.

Casolini P, Cigliana G, Alema GS, Ruggieri V, Angelucci L, Catalani A (1997) Effect of increased maternal corticosterone during lactation on hippocampal corticosteroid receptors, stress response and learning in offspring in the early stages of life. Neuroscience 79:1005-1012.

Catalani A, Marinelli M, Scaccianoce S, Nicolai R, Muscolo LAA, Porcu A, Korányi L, Piazza PV, Angelucci L (1993) Progeny of mothers drinking corticosterone during lactation has lower stress-induced corticosterone secretion and better cognitive performance. Brain Res 624:209-215.

Catalani A, Casolini P, Scaccianoce S, Patacchioli FR, Spinozzi P, Angelucci L (2000) Maternal corticosterone during lactation permanently affects brain corticosteroid receptors, stress response and behaviour in rat progeny. Neuroscience 100:319-325.

Catalani A, Casolini P, Cigliana G, Scaccianoce S, Consoli C, Cinque C, Zuena AR, Angelucci L (2002) Maternal corticosterone influences behavior, stress response and corticosteroid receptors in the female rat. Pharmacol Biochem Behav 73:105-114.

Choi SH, Lee DY, Kim SU, Jin BK (2005) Thrombin-induced oxidative stress contributes to the death of hippocampal neurons in vivo: role of microglial NADPH oxidase. J Neurosci 25:4082-4090.

Cinque C, Zuena AR, Casolini P, Ngomba RT, Melchiorri D, Maccari S, Nicoletti F, Di Giorgi G, Catalani A (2003) Reduced activity of hippocampal group-I metabotropic glutamate receptors in learning-prone rats. Neuroscience 122:277-284.

De Kloet ER, Vreugdenhil E, Oitzl MS, Joels M (1998) Brain corticosteroid receptor balance in health and disease Endocr Rev 19:269-301.

De Kloet ER, Derijk RH, Meijer OC (2007) Therapy Insight: is there an imbalanced response of mineralocorticoid and glucocorticoid receptors in depression? Nat Clin Pract Endocrinol Metab 3:168-179.

De Vry J, Horvath E, Schreiber R (2001) Neuroprotective and behavioral effects of the selective metabotropic glutamate $\mathrm{mGlu}(1)$ receptor antagonist BAY 36-7620. Eur J Pharmacol 428:203-214.

Felszeghy K, Gaspar E, Nyakas C (1996) Long-term selective downregulation of brain glucocorticoid receptors after neonatal dexamethasone treatment in rats. J Neuroendocrinol 8:493-499.

Ferrand-Drake M, Wieloch T (1999) The time-course of DNA fragmentation in the choroid plexus and the CAl region following transient global ischemia in the rat brain. The effect of intra-ischemic hypothermia. Neuroscience 93:537-549.

Hoffman GE, Merchenthaler I, Zup SL (2006) Neuroprotection by ovarian hormones in animal models of neurological disease. Endocrine 29:217-231.

Kaffman A, Meaney MJ (2007) Neurodevelopmental sequelae of postnatal maternal care in rodents: clinical and research implications of molecular insights. J Child Psychol Psychiatry 48:224-244.

Kamphuis PJ, Bakker JM, Broekhoven MH, Kunne C, Croiset G, Lentjes EG, Tilders FJ, van Bel F, Wiegant VM (2002) Enhanced glucocorticoid feedback inhibition of hypothalamo-pituitary-adrenal responses to stress in adult rats neonatally treated with dexamethasone. Neuroendocrinology 76:158-169.

Koide T, Wieloch TW, Siesjo BK (1986) Chronic dexamethasone pretreatment aggravates ischemic neuronal necrosis. J Cereb Blood Flow Metab 6:395-404.

Liu D, Diorio J, Tannenbaum B, Caldji C, Francis D, Freedman A, Sharma
Shakti, Pearson D, Plotsky PM, Meaney MJ (1997) Maternal care, hippocampal glucocorticoid receptors, and hypothalamic-pituitary-adrenal responses to stress. Science 277:1659-1662.

Liu Y, Imai H, Sadamatsu M, Tsunashima K, Kato N (2005) Cytokines participate in neuronal death induced by trimethyltin in the rat hippocampus via type II glucocorticoid receptors. Neurosci Res 51:319-327.

Macleod MR, Johansson IM, Soderstrom I, Lai M, Gido G, Wieloch T, Seckl JR, Olsson T (2003) Mineralocorticoid receptor expression and increased survival following neuronal injury. Eur J Neurosci 17:1549-1555.

McEwen BS (1999) Stress and hippocampal plasticity. Annu Rev Neurosci 22:105-122.

Meaney MJ, Szyf M (2005) Maternal care as a model for experiencedependent chromatin plasticity? Trends Neurosci 28:456-463.

Meaney MJ, Aitken DH, Sapolsky RM (1987) Thyroid hormones influence the development of hippocampal glucocorticoid receptors in the rat: a mechanism for the effects of postnatal handling on the development of the adrenocortical stress response. Neuroendocrinology 45:278-283.

Meaney MJ, Aitken DH, van Berkel C, Bhatnagar S, Sapolsky RM (1988) Effect of neonatal handling on age-related impairments associated with the hippocampus. Science 239:766-768.

Meaney MJ, Aitken DH, Sharma S, Viau V (1992) Basal ACTH, corticosterone and corticosterone-binding globulin levels over the diurnal cycle, and age-related changes in hippocampal type I and type II corticosteroid receptor binding capacity in young and aged, handled and nonhandled rats. Neuroendocrinology 55:204-213.

Moles A, Rizzi R, D’Amato FR (2004) Postnatal stress in mice: does "stressing" the mother have the same effect as "stressing" the pups? Dev Psychobiol 44:230-237.

Moroni F, Attucci S, Cozzi A, Meli E, Picca R, Scheideler MA, Pellicciari R, Noe C, Sarichelou I, Pellegrini-Giampietro DE (2002) The novel and systemically active metabotropic glutamate 1 (mGlul) receptor antagonist 3-MATIDA reduces post-ischemic neuronal death. Neuropharmacology 42:741-751.

Pellegrini-Giampietro DE (2003) The distinct role of mGlul receptors in post-ischemic neuronal death. Trends Pharmacol Sci 24:461-470.

Rees SL, Panesar S, Steiner M, Fleming AS (2004) The effects of adrenalectomy and corticosterone replacement on maternal behavior in the postpartum rat. Horm Behav 46:411-419.

Sapolsky RM (1986) Glucocorticoid toxicity in the hippocampus: reversal by supplementation with brain fuels. J Neurosci 6:2240-2244.

Sapolsky RM, Meaney MJ (1986) Maturation of the adrenocortical stress response: neuroendocrine control mechanisms and the stress hyporesponsive period. Brain Res 396:64-76.

Sapolsky RM, Pulsinelli WA (1985) Glucocorticoids potentiate ischemic injury to neurons: therapeutic implications. Science 229:1397-1400.

Shinwell ES, Karplus M, Reich D, Weintraub Z, Blazer S, Bader D, Yurman S, Dolfin T, Kogan A, Dollberg S, Arbel E, Goldberg M, Gur I, Naor N, Sirota L, Mogilner S, Zaritsky A, Barak M, Gottfried E (2000) Early postnatal dexamethasone treatment and increased incidence of cerebral palsy. Arch Dis Child 83:F177-F181.

Vallèe M, Mayo W, Dellu F, Le Moal M, Simon H, Maccari S (1997) Prenatal stress induces high anxiety and postnatal handling induces low anxiety in adult offspring: correlation with stress-induced corticosterone secretion. J Neurosci 17:2626-2636.

Weaver IC, La Plante P, Weaver S, Parent A, Sharma S, Diorio J, Chapman KE, Seckl JR, Szyf M, Meaney MJ (2001) Early environmental regulation of hippocampal glucocorticoid receptor gene expression: characterization of intracellular mediators and potential genomic target sites. Mol Cell Endocrinol 185:205-218.

Weaver IC, Cervoni N, Champagne FA, D’Alessio AC, Sharma S, Seckl JR, Dymov S, Szyf M, Meaney MJ (2004) Epigenetic programming by maternal behavior. Nat Neurosci 7:847-854. 